\title{
Nomogram for predicting the overall survival of the patients with oesophageal signet ring cell carcinoma
}

\author{
Feng Wang^, Shu-Geng Gao^, Qi Xue^, Feng-Wei Tan^, Yu-Shun Gao^, Da-Li Wang^, \\ You-Sheng Mao^, Jun Zhao^, Yin Li^, Xiang-Yang Yu^, Hong Cheng^, Chen-Guang Zhao^, \\ Ding Yang^, Ju-Wei $\mathrm{Mu}^{\wedge}$
}

Department of Thoracic Surgery, National Cancer Center/National Clinical Research Center for Cancer/Cancer Hospital, Chinese Academy of Medical Sciences and Peking Union Medical College, Beijing, China

Contributions: (I) Conception and design: F Wang; (II) Administrative support: JW Mu, SG Gao, Q Xue, FW Tan, YS Gao, DL Wang, YS Mao, J Zhao, Y Li; (III) Provision of study materials or patients: F Wang, JW Mu; (IV) Collection and assembly of data: F Wang, CG Zhao, D Yang; (V) Data analysis and interpretation: F Wang, XY Yu, H Cheng; (VI) Manuscript writing: All authors; (VII) Final approval of manuscript: All authors.

Correspondence to: Ju-wei Mu, MD. Professor, Department of Thoracic Surgery, National Cancer Center/National Clinical Research Center for Cancer/ Cancer Hospital, Chinese Academy of Medical Sciences and Peking Union Medical College, Beijing 100021, China. Email: mujuwei@cicams.ac.cn.

Background: The purpose of this study was to explore the prognostic factors of oesophageal signet ring cell (SRC) carcinoma and to construct a nomogram for predicting the outcome of SRC carcinoma of oesophagus.

Methods: A total of 968 cases of oesophageal SRC carcinoma were extracted from the Surveillance, Epidemiology, and End Results (SEER) database between 2004 and 2016. Cases were divided into training cohort and validation cohort. Univariate and multivariable Cox analyses was performed to select the predictors of overall survival (OS for the nomogram. The performance of nomogram was validated with Harrell's concordance index (C-index), calibration curves and decision curve analysis (DCA).

Results: The 1 - and 5-year OS in the training cohort were 0.446 and 0.146 , respectively, and the 1and 5 -year OS in the validation cohort were 0.459 and 0.138 . The independent prognostic factors for establishing the nomogram were marital status, invasion of the surrounding tissue, lymph node metastasis, distant metastasis, surgery and chemotherapy. The Harrell's c-index value of the training cohort and validation cohort were 0.723 and 0.708 . In the calibration curves, the predicted survival probability and the actual survival probability have a considerable consistency. DCA indicated the favourable potential clinical utility of the nomogram.

Conclusions: A nomogram to predict the OS of patients with oesophageal SRC carcinoma was established. The validation of the nomogram fully demonstrates its great performance.

Keywords: Oesophageal cancer; signet ring cell (SRC); nomogram

Submitted Oct 15, 2020. Accepted for publication Dec 31, 2020.

doi: $10.21037 /$ jtd-20-3084

View this article at: http://dx.doi.org/10.21037/jtd-20-3084

\footnotetext{
^ ORCID: Feng Wang, 0000-0002-3391-0241; Shu-Geng Gao, 0000-0003-0947-4926; Qi Xue, 0000-0002-5086-4654; Feng-Wei Tan, 0000-0002-8210-684X; Yu-Shun Gao, 0000-0002-1997-3115; Da-Li Wang, 0000-0002-5096-2895; You-Sheng Mao, 0000-0002-46407389; Jun Zhao, 0000-0002-9580-5439; Yin Li, 0000-0003-0033-808X; Xiang-Yang Yu, 0000-0002-2372-8905; Hong Cheng, 0000-00025286-5466; Chen-Guang Zhao, 0000-0003-3205-9384; Ding Yang, 0000-0001-7749-8255; Ju-Wei Mu, 0000-0002-9917-2325.
} 


\section{Introduction}

Oesophageal cancer is the 9th most common cancer and the 6th most common cause of cancer death globally (1). The main pathological types of oesophageal cancer are adenocarcinoma and squamous cell carcinoma. Squamous cell carcinoma is the predominant pathological type of oesophageal carcinoma worldwide. However, in the USA, Australia and some European countries, the incidence of oesophageal adenocarcinoma now exceeds that of oesophageal squamous cell carcinoma $(2,3)$.

As a special pathological type of adenocarcinoma, signet ring cell (SRC) carcinoma is characterized by the appearance of a large vacuole containing mucin that squeezes the nucleus to the periphery of the cancer cell, making the cell look like a signet ring (4). SRC carcinoma can be diagnosed if more than $50 \%$ of tumours contain SRCs, according to the World Health Organization (WHO) criteria (5). SRC carcinoma is a rare pathological type of oesophageal cancer, and only approximately $3.5 \%$ to $5 \%$ of oesophageal cancers are SRC carcinomas (6-8). The first article to describe SRC carcinoma in the oesophagus was published in 1978 (9). SRCs have been found in colorectal cancer, prostate cancer, bladder cancer, breast cancer, gastric cancer and other adenocarcinomas. Various studies have shown that SRC carcinoma is an aggressive adenocarcinoma, and its presence suggests a poor prognosis (8,10-12). At present, most studies on SRC carcinoma are focused on gastric cancer and colorectal cancer, and there are relatively few studies on SRC carcinoma of the oesophagus.

A nomogram is also known as an alignment diagram. Based on multivariate regression analysis, a nomogram integrates multiple prediction indicators and then uses line segments with scales to draw them on the same plane in a certain proportion to express the predictive value of each variable in the prediction model. A nomogram is a convenient model for predicting clinical events, which is helpful in individualized treatment, clinical decision making and clinical trial design. Most of the current nomograms are for localized or metastatic oesophageal squamous cell carcinoma or adenocarcinoma, and no studies have established a predictive model for oesophageal SRC carcinoma (13-16). Therefore, the purpose of our study was to construct and validate a novel nomogram for predicting the outcomes of oesophageal SRC carcinoma patients using data from the Surveillance, Epidemiology, and End Results (SEER) database.
We present the following article in accordance with the TRIPOD reporting checklist (available at http://dx.doi. org/10.21037/jtd-20-3084).

\section{Methods}

\section{Patient selection}

All of the cases in this study were from the SEER database (the SEER 18 registries database with the additional treatment field, released in April 2019, www.seer.cancer. gov). The study was conducted in accordance with the Declaration of Helsinki (as revised in 2013). The SEER database, which includes approximately $30 \%$ of the United States population, is supported by the National Cancer Institute. The SEER*Stat 8.3.6 software was installed to extract the information of patients with oesophageal SRC carcinoma diagnosed between 2004 and 2016. We limited the period to 2004-2016, as detailed information about the TNM stage (AJCC $6^{\text {th }}$ edition) and distant metastasis is only available from 2004. The selection criteria were as follows: (I) the site recorded was the oesophagus; (II) the histology code was $8490 / 3$ SRC carcinoma; and (III) the year of diagnosis was 2004-2016. The exclusion criteria were (I) cases without race information; (II) cases without accurate TNM stage information. Ultimately, 968 cases were enrolled in our study cohort (Figure 1).

\section{Outcome}

The variables extracted from the SEER database included age, sex, race, marital status, primary site, $\mathrm{T}$ stage, $\mathrm{N}$ stage, $M$ stage (the TNM staging of some patients undergoing surgery were pathological staging, while the others were clinical staging), grade, surgery, chemotherapy, radiation sequence with surgery, total number of malignant tumours, cause of death, survival time, and vital status. Patient deaths from all causes were regarded as uncensored cases for the overall survival (OS) analysis.

Patients were divided into 2 groups based on age $(\leq 75$, $>75$ years). Marital status was reclassified as married and single/unknown (divorced, separated, single, widowed, unknown). According to the 6th edition of the Union for International Cancer Control-American Joint Committee on Cancer (UICC-AJCC), tumour, node, metastasis (TNM) staging system, and $\mathrm{T}$ stage were reclassified into 2 groups (no invasion of the surrounding tissue: T1-T3; invasion of the surrounding tissue: $\mathrm{T} 4)$. The $\mathrm{N}$ stage was converted into 


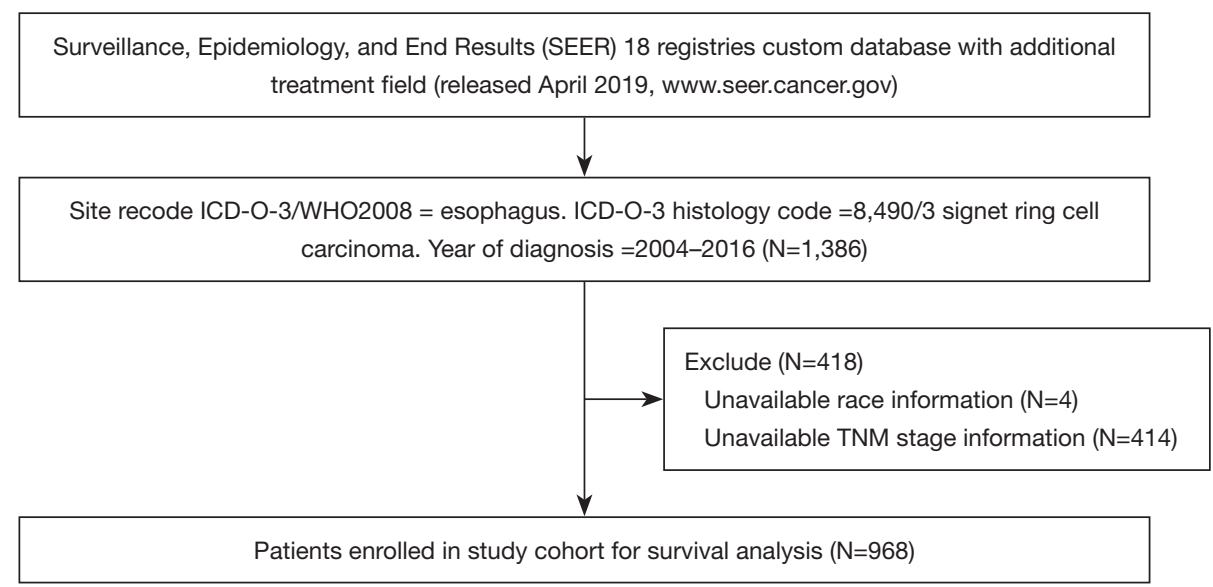

Figure 1 Study diagram of the selection process. ICD-O-3, 3rd edition of the International Classification Disease for Oncology; WHO, World Health Organization.

a variable named lymph node metastasis (yes: N1, no: N0). The $M$ stage was converted into a variable named distant metastasis (yes: M1, no: M0). Surgery was reclassified into 3 groups (no surgery: SEER codes 00; local destruction or excision: SEER codes 10-14, 20-27; surgery: SEER codes 30, 40, 50-55, 80). Radiation sequences with surgery were converted into a variable named radiation therapy (yes, no).

All enrolled cases were divided into a training cohort and a validation cohort at a ratio of 7:3 randomly. The training cohort was used to establish the nomogram, and the validation cohort was used to validate the nomogram.

\section{Statistical analysis}

The clinicopathological features between the training and validation cohorts were compared by the using Chisquare $\left(\chi^{2}\right)$ test. OS was calculated using the Kaplan-Meier method. The Cox proportional hazard regression model was used for univariate and multivariate analyses. Variables with statistical significance $(\mathrm{P}<0.1)$ according to univariate analysis were included in the multivariate analysis. SPSS software (version 25.0 IBM, USA) was used for statistical analysis.

By using R software (version 4.0.0 R Foundation, Austria), we constructed a nomogram using the variables selected from the training cohort. Harrell's c-index was used to evaluate the predictive accuracy of the nomogram. Calibration curves were generated to visualize the discrimination between predicted and actual OS. The nomogram, Harrell's c-index and calibration curve were produced using the "rms" package of R software.
The decision curve analysis (DCA), a novel diagram for evaluating the prediction model, was used to estimate the clinical utility of the nomogram $(17,18)$.

\section{Results}

\section{Baseline characteristics}

A total of 968 eligible cases were enrolled in this study cohort and were randomly divided into the training cohort (677 cases) and validation cohort (291 cases) at a ratio of $7: 3$. The characteristics of the oesophageal SRC carcinoma patients in the training cohort and validation cohort are shown in Table 1. In all of the enrolled cases, most of the patients were younger than 75 years old, accounting for approximately $76.9 \%$ of the total patients. Approximately $86.9 \%$ of the patients were male. White and married people represented the majority of the patients.

Since SRC carcinoma is a type of adenocarcinoma, most (85.2\%) of the tumours were located in the lower third of the oesophagus. As SRC carcinoma is a highly malignant tumour, most tumours were found to have a high grade $(\mathrm{III}+\mathrm{IV})$. At the same time, there were more cases with lymph node metastasis than without, and approximately $15.5 \%$ of the patients had invasion of the surrounding tissue, while $30.1 \%$ of the patients had distant metastases.

In terms of treatment, $34.8 \%$ of patients received surgical treatment, $27.5 \%$ received radiotherapy, and $71.3 \%$ received chemotherapy. Approximately $22.3 \%$ of the patients were diagnosed with two or more malignant tumours. The Chi-square $\left(\chi^{2}\right)$ test was performed to 
Table 1 Characteristics of oesophageal SRC carcinoma patients in the training cohort and validation cohort

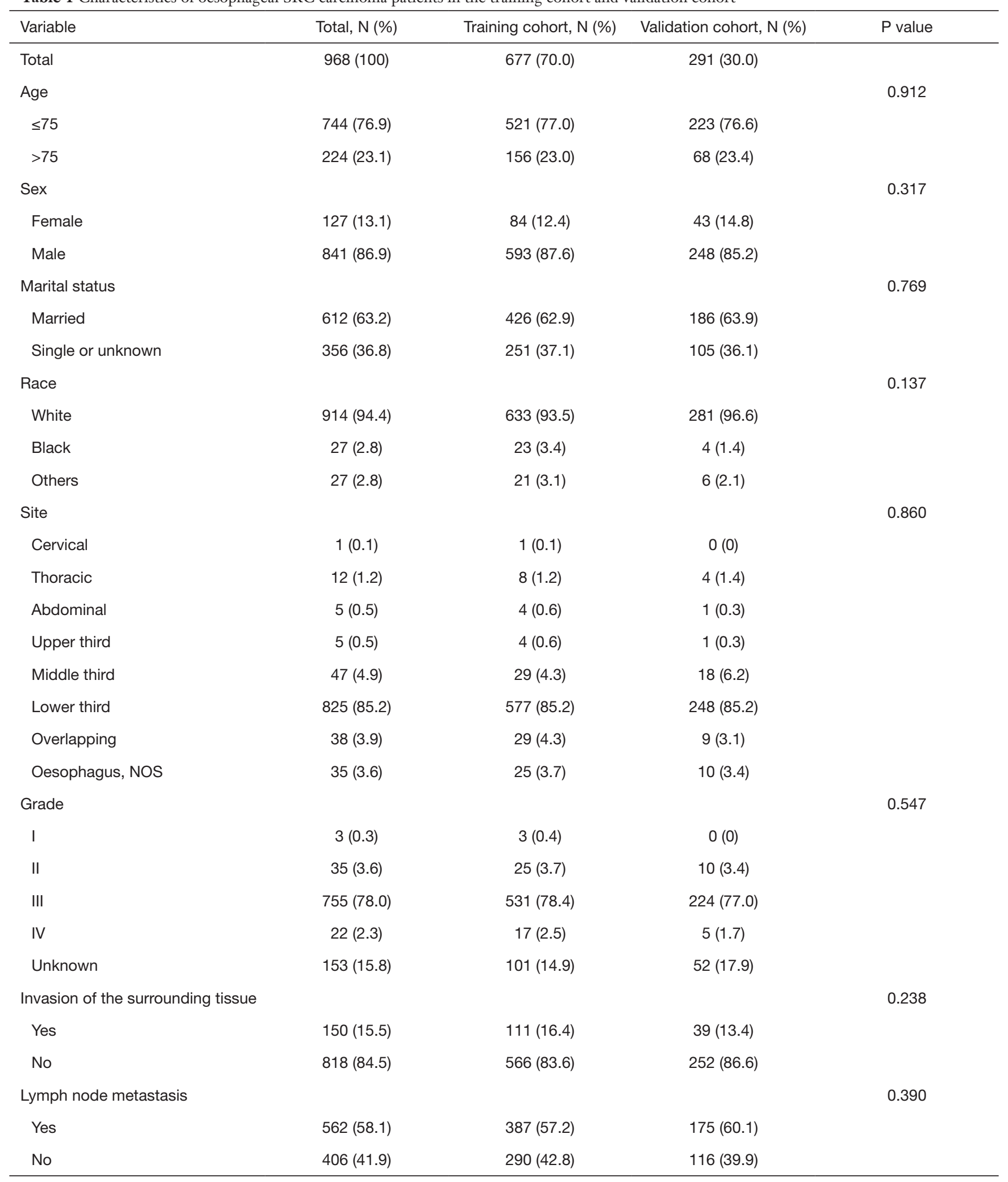

Table 1 (continued) 
Table 1 (continued)

\begin{tabular}{|c|c|c|c|c|}
\hline Variable & Total, N (\%) & Training cohort, N (\%) & Validation cohort, N (\%) & $P$ value \\
\hline Yes & $291(30.1)$ & $210(31.0)$ & $81(27.8)$ & \\
\hline No & $677(69.9)$ & $467(69.0)$ & $210(72.2)$ & \\
\hline Surgery & & & & 0.223 \\
\hline Local destruction or excision & $24(2.5)$ & $13(1.9)$ & $11(3.8)$ & \\
\hline Surgery & $337(34.8)$ & $235(34.7)$ & $102(35.1)$ & \\
\hline Radiation therapy & & & & 0.634 \\
\hline Yes & $266(27.5)$ & $183(27.0)$ & $83(28.5)$ & \\
\hline Yes & $690(71.3)$ & $479(70.8)$ & $211(72.5)$ & \\
\hline No or unknown & $278(28.7)$ & $198(29.2)$ & $80(27.5)$ & \\
\hline Number of malignant tumours & & & & 0.243 \\
\hline$=1$ & $752(77.7)$ & $519(76.7)$ & $233(80.1)$ & \\
\hline$\geq 2$ & $216(22.3)$ & $158(23.3)$ & $58(19.9)$ & \\
\hline
\end{tabular}

compare categorical variables between groups, but there was no variable with a $\mathrm{P}$ value less than 0.05 .

The survival curves of the training cohort and the validation cohort generated using the Kaplan-Meier method are shown in Figure 2. The 1- and 5-year OS in the training cohort were 0.446 and 0.146 , respectively, and the 1 - and 5 -year OS in the validation cohort were 0.459 and 0.138 .

\section{Prognostic factors for oesophageal SRC patients}

A Cox proportional hazards regression model was applied for univariate and multivariate analyses of OS. As shown in Table 2, according to the univariate analysis, a total of 8 variables (age, marital status, invasion of the surrounding tissue, lymph node metastasis, distant metastasis, surgery, radiation therapy, and chemotherapy) were considered to be statistically significant $(\mathrm{P}<0.1)$ and could be included in the multivariate analysis. Before performing the multivariate analysis, a multicollinearity diagnosis was conducted for these 8 variables. The variance inflation factor (VIF) values for age, marital status, invasion of the surrounding tissue, lymph node metastasis, distant metastasis, surgery, radiation therapy, and chemotherapy were $1.135,1.036,1.071,1.080,1.174,2.520,2.569$, and 1.270 , respectively. We found that the VIF value for radiation therapy was the largest, indicating the existence of a multicollinearity problem.

After removing the variable radiation therapy, multicollinearity diagnosis was performed again, and it was found that none of the variables indicated a significant problem with multicollinearity, with all the VIF values less than 2 (age, 1.135; marital status, 1.033; invasion of the surrounding tissue, 1.071; lymph node metastasis, 1.080; distant metastasis, 1.171, surgery, 1.203; and chemotherapy, 1.080). These 7 variables were thus included in the multivariate Cox regression analysis.

As shown in Table 3, the $\mathrm{P}$ value of age was 0.901 , which was considered to have no statistical significance. Finally, the independent prognostic factors for establishing the nomogram were chosen, and the nomogram was based on marital status, invasion of the surrounding tissue, lymph node metastasis, distant metastasis, surgery and chemotherapy.

\section{Development and validation of the nomogram}

Based on the six variables described above (marital 

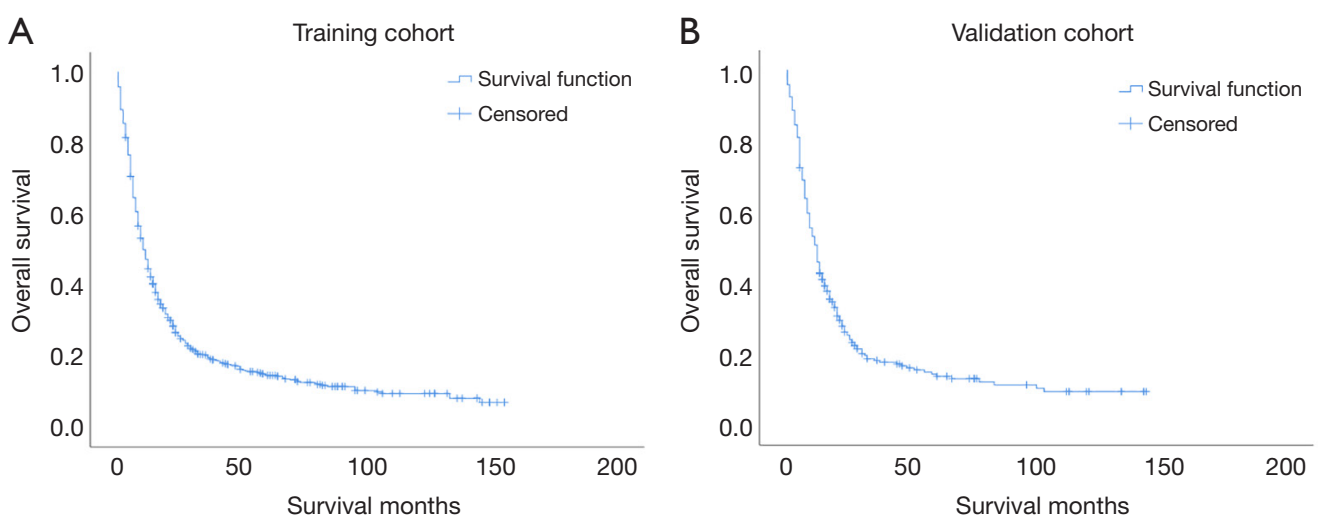

Figure 2 Kaplan-Meier survival curves of the overall survival (OS) for oesophageal signet ring carcinoma patients. (A) Training cohort; (B) validation cohort.

Table 2 Univariate cox analysis of overall survival in the training cohort

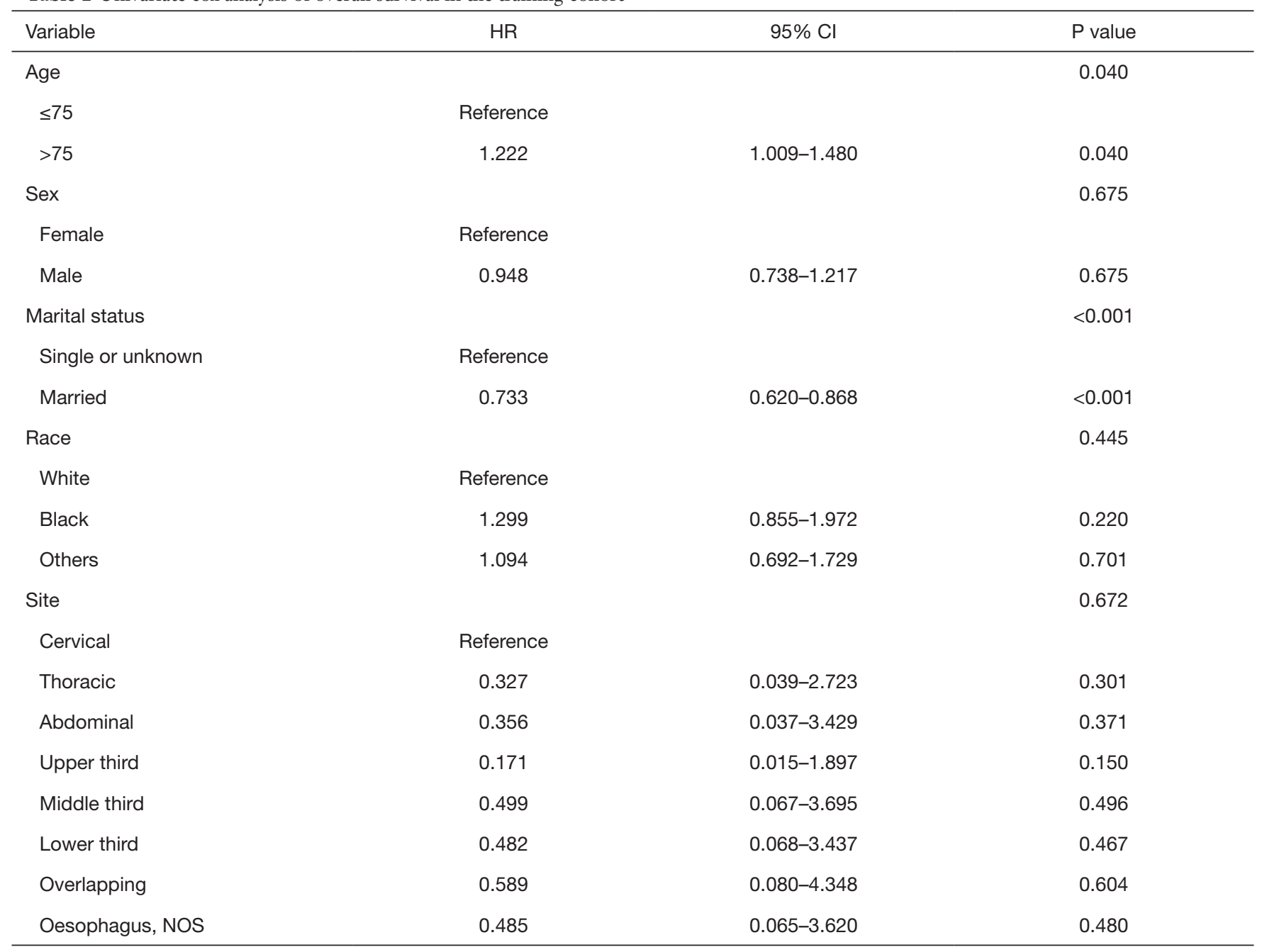

Table 2 (continued) 
Table 2 (continued)

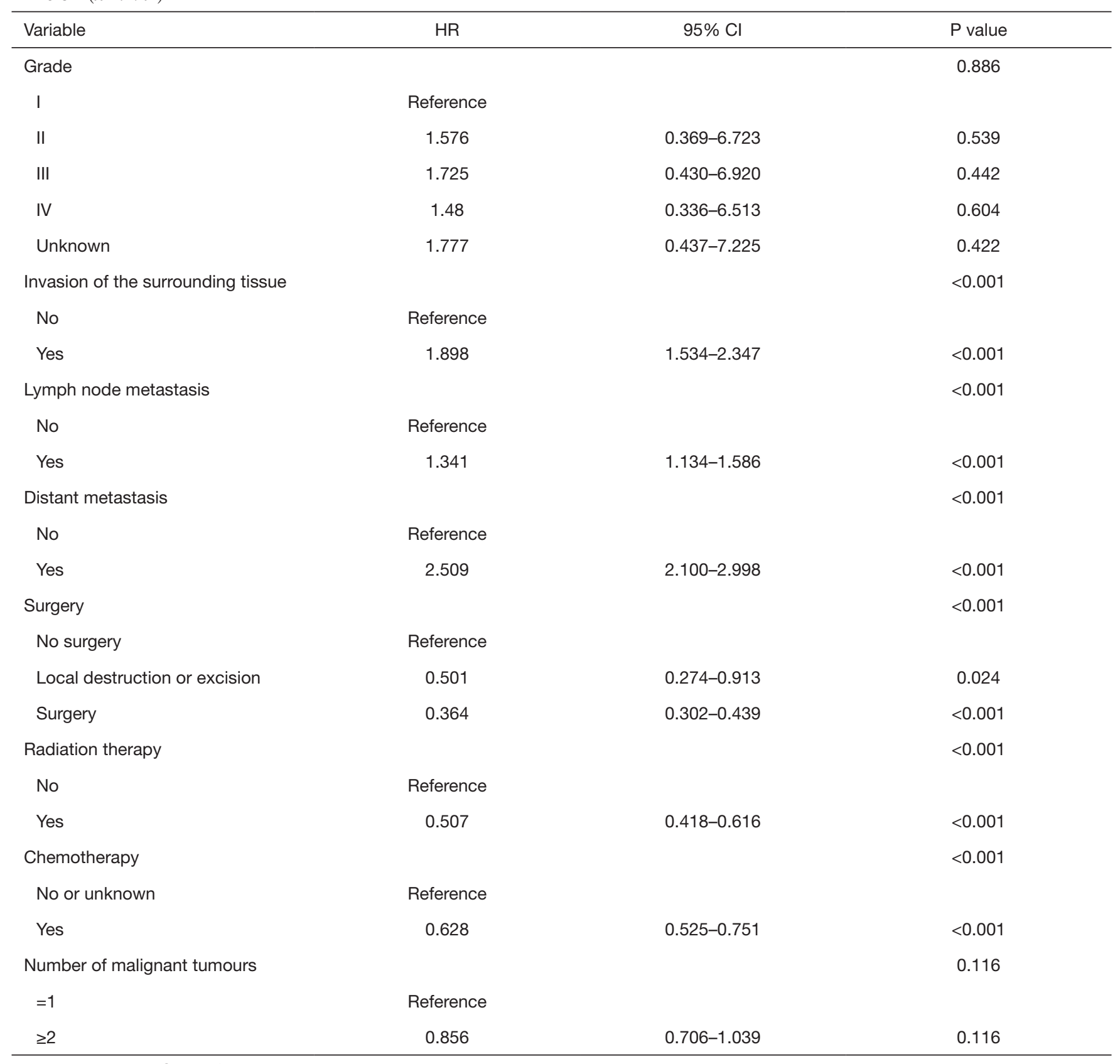

$\mathrm{HR}$, hazard ratio; $\mathrm{Cl}$, confidence interval.

status, invasion of the surrounding tissue, lymph node metastasis, distant metastasis, surgery and chemotherapy), a nomogram was constructed to predict the 1-year, 3 -year and 5-year OS of patients with oesophageal SRC carcinoma, as shown in Figure 3. In the nomogram, surgery had the greatest influence on prognosis, while marital status had the least influence. By adding the scores of each item to obtain the total score, the corresponding survival probability was obtained from the nomogram. The Harrell's c-index value of the training cohort and validation cohort were 0.723 and 0.708 , respectively. As shown in Figure 4, by drawing the calibration curves (200 bootstrap resamples), it can be seen that the predicted survival probability and the actual survival probability have 
Table 3 Multivariate cox analysis of overall survival in the training cohort

\begin{tabular}{|c|c|c|c|}
\hline Variable & HR & $95 \% \mathrm{Cl}$ & $P$ value \\
\hline$\leq 75$ & Reference & & \\
\hline$>75$ & 0.987 & $0.805-1.211$ & 0.901 \\
\hline Marital status & & & 0.090 \\
\hline Married & 0.862 & $0.726-1.024$ & 0.090 \\
\hline Invasion of the surrounding tissue & & & $<0.001$ \\
\hline No & Reference & & \\
\hline Yes & 1.431 & $1.147-1.784$ & $<0.001$ \\
\hline Yes & 1.446 & $1.214-1.723$ & $<0.001$ \\
\hline Distant metastasis & & & $<0.001$ \\
\hline No & Reference & & \\
\hline Yes & 1.882 & $1.553-2.281$ & $<0.001$ \\
\hline Surgery & & & $<0.001$ \\
\hline No surgery & Reference & & \\
\hline Local destruction or excision & 0.516 & $0.279-0.953$ & 0.034 \\
\hline Surgery & 0.419 & $0.342-0.514$ & $<0.001$ \\
\hline
\end{tabular}

HR, hazard ratio; $\mathrm{Cl}$, confidence interval.

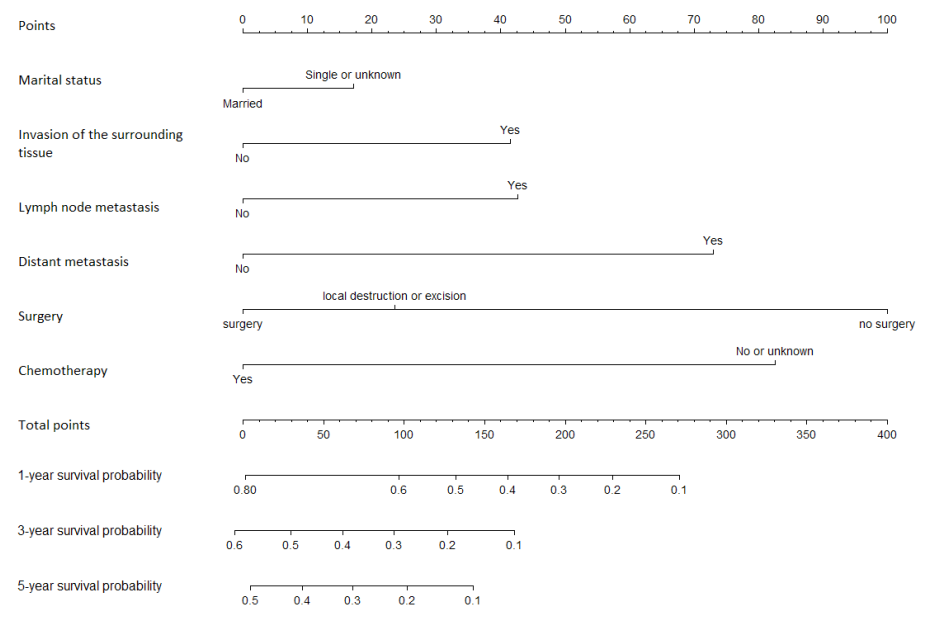

Figure 3 Nomogram for predicting 1-, 3- and 5-year overall survival of patients with oesophageal signet ring cell carcinoma. 

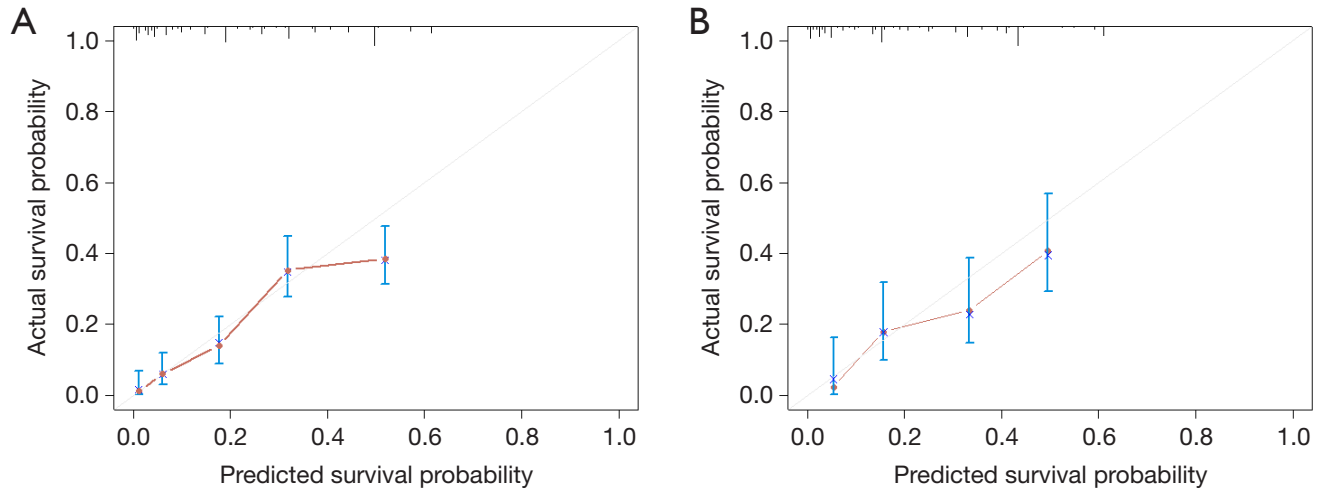

Figure 4 Calibration curves for predicting 3-year overall survival in the training cohort (A) and validation cohort (B).
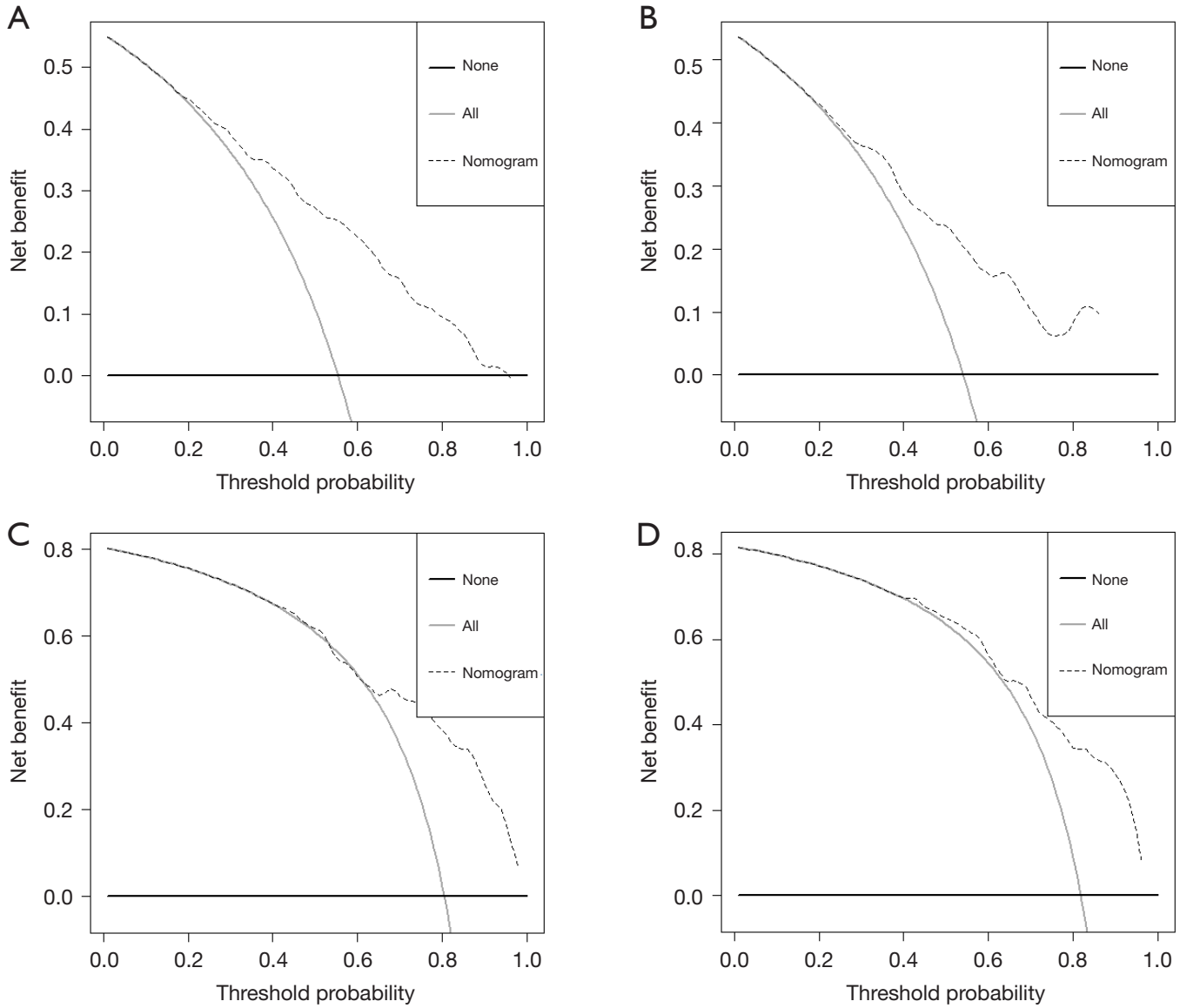

Figure 5 Decision curve analysis of the nomogram for predicting overall survival at 1-year point in the trainning cohort (A) and validation cohort (B) and overall survival at 3-year point in the trainning cohort (C) and validation cohort (D).

a considerable consistency.

Finally, as shown in Figure 5, we performed DCA to evaluate the clinical utility of the predictive model. The $\mathrm{x}$-axis represents the threshold probabilities, and the $\mathrm{y}$-axis measures the net benefit calculated by adding the true positives and subtracting the false positives. The horizontal 
line assumes that death occurred in no patients, whereas the gray line assumes that all patients will have death at a specific threshold probability. The dashed line represents the net benefit of using the nomogram. As we can see in the DCA, in the range $0-1.0$ of threshold probabilities nomogram showed better net benefit than the other two extreme cases. The DCA showed strong positive net benefits in the nomogram among wide ranges of the threshold probabilities, indicating the favourable potential clinical utility of the nomogram.

\section{Discussion}

Oesophageal SRC carcinoma is a rare pathological type of oesophageal adenocarcinoma. According to previous studies, SRC carcinoma of the oesophagus is a malignant tumour with a poor prognosis (7-9). There are few studies on the prognostic factors of SRC carcinoma of the oesophagus, and no prognostic model of oesophageal SRC carcinoma has been developed, which makes it difficult to predict the outcomes of patients with oesophageal SRC. In this paper, the first predictive model for oesophageal SRC carcinoma was constructed using the SEER database, and subsequent validation of the model established its great performance in predicting the outcome of oesophageal SRC carcinoma.

Nomograms, as clinical prediction models, have been widely used to predict the outcomes of cancer patients. Several predictive models are currently available for oesophageal cancer $(13,14,16,19,20)$, but none of them focus on SRC carcinoma of the oesophagus. Current studies on the prognosis of SRC of the oesophagus suggest that female sex, unmarried, invasion of adjacent organs, a high tumour grade, metastasis of regional lymph nodes or distant organs and no chemotherapy were independent prognostic factors $(21,22)$. However, the sample sizes of these studies were small and some prognostic factors associated with oesophageal SRC were found, but no predictive models were further constructed and validated.

In this study, nearly 1,000 cases with oesophageal SRC carcinoma from the SEER database were enrolled, and they were randomized into training and validation cohorts to validate the predictive model. Univariate and multivariate analyses of the existing variables in the SEER database were performed. Finally, six variables, including marital status, invasion of the surrounding tissue, lymph node metastasis, distant metastasis, surgery and chemotherapy, were selected as independent prognostic factors for construction of the nomogram.
According to the predictive model constructed in this study, surgery and chemotherapy had the greatest impact on prognosis, which also supports the concept that surgery and chemotherapy are currently the most important treatment methods for oesophageal SRC. This conclusion is consistent with previous studies on oesophageal cancer (23-26). For radiotherapy, this variable was removed in this study due to the collinearity problem. We think the collinearity problem may be related to the fact that most patients receiving radiotherapy are patients in an advanced stage with no chance of surgery. There is a significant correlation between whether a patient receives radiotherapy and the TNM stage of the patient, so the effect of radiotherapy on prognosis will be interfered with by the TNM stage of the patient. In addition, as SRC of the oesophagus is a type of adenocarcinoma and the sensitivity of oesophageal adenocarcinoma to radiotherapy is significantly lower than that of oesophageal squamous cell carcinoma (27), radiotherapy was not included in the final prediction model.

According to our study, invasion of the surrounding tissue, lymph node metastasis, and distant metastasis can all adversely affect the prognosis of patients. The presence of surrounding tissue invasion and lymph node or distant organ metastasis all indicate an advanced TNM stage, so these patients have a worse prognosis, which is consistent with the majority of existing research results. However, there are still some controversial studies on oesophageal adenocarcinoma. Agoston and colleagues found that the $\mathrm{T}$ stage and $\mathrm{N}$ stage had no effect on the prognosis of oesophageal adenocarcinoma (28). Further studies are expected to clarify the relationship between these factors and the prognosis of SRC of the oesophagus.

In this study, marital status was also found to be a prognostic factor. As seen in the nomogram, single patients had a worse prognosis. The effect of marital status on the prognosis of patients with malignant tumours has been mentioned in many previous retrospective studies. Studies of colorectal cancer, breast cancer and prostate cancer have shown that married patients exhibit better survival than unmarried patients $(29,30)$. The reason for this difference may be that marital status affects a patients' mood and quality of life $(31,32)$. More prospective studies are expected to confirm the relationship between marital status and prognosis in patients with oesophageal SRC.

There are still some limitations in this study. First, this study is a retrospective study, and selection bias is inevitable. Second, since the data in this study were obtained from the SEER database, some information that may impact the 
prognosis were not included, including the proportion of SRCs in tumour cells, comorbidities, the chemotherapy regimen, etc. Moreover, chemotherapy and radiation therapy data are categorized as either "yes-patient had chemotherapy or radiation therapy" or "no/unknown-no evidence of chemotherapy or radiation therapy was found in the medical records examined, the biases associated with these unmeasured reasons affect analyses Third, due to the low incidence of SRC carcinoma of the oesophagus, sufficient external data were not available for validation in this study. In the future, it is expected that more prospective multicentre large studies will be conducted on oesophageal SRC carcinoma to improve our diagnosis and treatment of SRC carcinoma of the oesophagus.

\section{Conclusions}

We built a nomogram to predict the OS of patients with oesophageal SRC carcinoma. The subsequent validation of the predictive model fully demonstrates its great performance. This model can be used to predict the outcomes of patients with oesophageal SRC carcinoma and provide guidance for their individualized treatment.

\section{Acknowledgments}

The authors acknowledge the tremendous effort made by the Surveillance, Epidemiology, and End Results (SEER) database program to create the SEER database.

Funding: Supported by the Capital Health Development Research Project, No. 2014-1-4021.

\section{Footnote}

Reporting Checklist: The authors have completed the TRIPOD reporting checklist. Available at http://dx.doi. org/10.21037/jtd-20-3084

Conflicts of Interest: All authors have completed the ICMJE uniform disclosure form (available at http://dx.doi. org/10.21037/jtd-20-3084). The authors have no conflicts of interest to declare.

Ethical Statement: The authors are accountable for all aspects of the work in ensuring that questions related to the accuracy or integrity of any part of the work are appropriately investigated and resolved. The study was conducted in accordance with the Declaration of Helsinki (as revised in 2013).

Open Access Statement: This is an Open Access article distributed in accordance with the Creative Commons Attribution-NonCommercial-NoDerivs 4.0 International License (CC BY-NC-ND 4.0), which permits the noncommercial replication and distribution of the article with the strict proviso that no changes or edits are made and the original work is properly cited (including links to both the formal publication through the relevant DOI and the license). See: https://creativecommons.org/licenses/by-nc-nd/4.0/.

\section{References}

1. Fitzmaurice C, Dicker D, Pain A, et al. The Global Burden of Cancer 2013. JAMA Oncol 2015;1:505-27.

2. Pohl H, Welch HG. The role of overdiagnosis and reclassification in the marked increase of esophageal adenocarcinoma incidence. J Natl Cancer Inst 2005;97:142-6.

3. Lepage C, Rachet B, Jooste V, et al. Continuing rapid increase in esophageal adenocarcinoma in England and Wales. Am J Gastroenterol 2008;103:2694-9.

4. Guerrero-Medrano J, Delgado R, Albores-Saavedra J. Signet-ring sinus histiocytosis: a reactive disorder that mimics metastatic adenocarcinoma. Cancer 1997;80:277-85.

5. Jass JR, Sobin LH, Watanabe H. The World Health Organization's histologic classification of gastrointestinal tumors. A commentary on the second edition. Cancer 1990;66:2162-7.

6. Enlow JM, Denlinger CE, Stroud MR, et al. Adenocarcinoma of the esophagus with signet ring cell features portends a poor prognosis. Ann Thorac Surg 2013;96:1927-32.

7. Yendamuri S, Huang M, Malhotra U, et al. Prognostic implications of signet ring cell histology in esophageal adenocarcinoma. Cancer 2013;119:3156-61.

8. Nafteux PR, Lerut TE, Villeneuve PJ, et al. Signet ring cells in esophageal and gastroesophageal junction carcinomas have a more aggressive biological behavior. Ann Surg 2014;260:1023-9.

9. Berenson MM, Riddell RH, Skinner DB, et al. Malignant transformation of esophageal columnar epithelium. Cancer 1978;41:554-61.

10. Tajiri K, Sudou T, Fujita F, et al. Clinicopathological and Corresponding Genetic Features of Colorectal Signet Ring Cell Carcinoma. Anticancer Res 2017;37:3817-23.

11. El Hussein S, Khader SN. Primary signet ring cell 
carcinoma of the pancreas: Cytopathology review of a rare entity. Diagn Cytopathol 2019;47:1314-20.

12. Machlowska J, Pucułek M, Sitarz M, et al. State of the art for gastric signet ring cell carcinoma: from classification, prognosis, and genomic characteristics to specified treatments. Cancer Manag Res 2019;11:2151-61.

13. Zhou Z, Zhang H, Xu Z, et al. Nomogram predicted survival of patients with adenocarcinoma of esophagogastric junction. World J Surg Oncol 2015;13:197.

14. Cao J, Yuan P, Wang L, et al. Clinical Nomogram for Predicting Survival of Esophageal Cancer Patients after Esophagectomy. Sci Rep 2016;6:26684.

15. Sun Y, Wang J, Li Y, et al. Nomograms to predict survival rates for esophageal cancer patients with malignant behaviors based on ICD-0-3. Future Oncol 2019;15:121-32.

16. Zhu C, You Y, Liu S, et al. A Nomogram to Predict Distant Metastasis for Patients with Esophageal Cancer. Oncol Res Treat 2020;43:2-9.

17. Vickers AJ, Cronin AM, Elkin EB, et al. Extensions to decision curve analysis, a novel method for evaluating diagnostic tests, prediction models and molecular markers. BMC Med Inform Decis Mak 2008;8:53.

18. Kerr KF, Brown MD, Zhu K, et al. Assessing the Clinical Impact of Risk Prediction Models With Decision Curves: Guidance for Correct Interpretation and Appropriate Use. J Clin Oncol 2016;34:2534-40.

19. Liu M, Wang C, Gao L, et al. A nomogram to predict long-time survival for patients with M1 diseases of esophageal cancer. J Cancer 2018;9:3986-90.

20. Tang X, Zhou X, Li Y, et al. A Novel Nomogram and Risk Classification System Predicting the Cancer-Specific Survival of Patients with Initially Diagnosed Metastatic Esophageal Cancer: A SEER-Based Study. Ann Surg Oncol 2019;26:321-8.

21. Chen L, Liu X, Gao L, et al. The clinicopathological features and prognosis of signet ring cell carcinoma of the esophagus: A 10-year retrospective study in China. PLoS One 2017;12:e0176637.

22. Wan Z, Huang Z, Chen L. Survival predictors associated with signet ring cell carcinoma of the esophagus (SRCCE): A population-based retrospective cohort study. PLoS One 2017;12:e181845.

23. Medical Research Council Oesophageal Cancer Working
Group. Surgical resection with or without preoperative chemotherapy in oesophageal cancer: a randomised controlled trial. Lancet 2002;359:1727-33.

24. Wu PC, Posner MC. The role of surgery in the management of oesophageal cancer. Lancet Oncol 2003;4:481-8.

25. Cunningham D, Allum WH, Stenning SP, et al. Perioperative chemotherapy versus surgery alone for resectable gastroesophageal cancer. $\mathrm{N}$ Engl J Med 2006;355:11-20.

26. Allum WH, Stenning SP, Bancewicz J, et al. Long-term results of a randomized trial of surgery with or without preoperative chemotherapy in esophageal cancer. J Clin Oncol 2009;27:5062-7.

27. Lagergren J, Smyth E, Cunningham D, et al. Oesophageal cancer. Lancet 2017;390:2383-96.

28. Agoston AT, Zheng Y, Bueno R, et al. Predictors of Disease Recurrence and Survival in Esophageal Adenocarcinomas With Complete Response to Neoadjuvant Therapy. Am J Surg Pathol 2015;39:1085-92.

29. Osborne C, Ostir GV, Du X, et al. The influence of marital status on the stage at diagnosis, treatment, and survival of older women with breast cancer. Breast Cancer Res Treat 2005;93:41-7.

30. Aizer AA, Chen MH, McCarthy EP, et al. Marital status and survival in patients with cancer. J Clin Oncol 2013;31:3869-76.

31. Byers TE, Wolf HJ, Bauer KR, et al. The impact of socioeconomic status on survival after cancer in the United States: findings from the National Program of Cancer Registries Patterns of Care Study. Cancer 2008;113:582-91.

32. Nipp RD, El-Jawahri A, Fishbein JN, et al. The relationship between coping strategies, quality of life, and mood in patients with incurable cancer. Cancer 2016;122:2110-6.

Cite this article as: Wang F, Gao SG, Xue Q, Tan FW, Gao YS, Wang DL, Mao YS, Zhao J, Li Y, Yu XY, Cheng H, Zhao CG, Yang D, Mu JW. Nomogram for predicting the overall survival of the patients with oesophageal signet ring cell carcinoma. J Thorac Dis 2021;13(3):1315-1326. doi: 10.21037/jtd20-3084 